\title{
Gonadotropines équines : purifications, spécificités, dosages immunologiques et utilisation chez la jument
}

\author{
S Hofferer, F Lecompte, T Magallon, E Palmer, Y Combarnous \\ INRA, physiologie reproduction et URA CNRS 1291, 37380 Nouzilly, France
}

(Reçu le 15 septembre 1991; accepté le 15 november 1991)

\begin{abstract}
Résumé - La maîtrise pharmacologique de la reproduction chez la jument nécessite l'utilisation de gonadotropines homologues pour éviter l'apparition d'une résistance immunologique. Dans un premier temps des extraits hypophysaires équins bruts contenant à la fois la $\mathrm{LH}$ et la FSH équines (eLH et eFSH) ont été utilisés mais ont donné des résultats décevants. Afin d'améliorer ces résultats, nous avons entrepris la mise au point d'une méthode de séparation, à l'échelle pilote, de eLH et eFSH par chromatographie d'interaction hydrophobe sur colonne de phényl-sépharose. Le rendement de cette technique permet la préparation à grande échelle des fractions eLH et eFSH pour injection chez la jument. Les activités biologiques des fractions eLH et eFSH ont été évaluées chez la jument et comparées à celles de l'extrait brut hypophysaire. Les fractions eLH et eFSH sont apparues aussi actives que l'extrait brut dans l'induction d'ovulation et la superovulation respectivement, indiquant que la méthode de purification n'altère pas ces hormones. L'utilisation des gonadotropines équines purifiées s'est avérée surtout intéressante par la plus grande homogénéité des réponses ovariennes et la meilleure synchronisation des ovulations obtenues.
\end{abstract}

gonadotropine / jument / ovulation / superovulation / purification

Summary - Equine gonadotropins: separation, immunoassays and use in ovulation induction and superovulation in mares. The pharmacological control of reproduction in the mare requires the use of equine gonadotrophins to avoid induced immunological resistance. Crude equine gonadotrophins (CEG) have been used for several years but the presence of both equine luteinizing hormone (eLH) and follicle-stimulating hormone (eFSH) in CEG has so far led to disappointing results in superovulation. In order to overcome this problem, the separation of eLH and eFSH activities from CEG appeared necessany. The hydrophobic properties of the 2 hormones were different enough to permit their efficient separation by hydrophobic interaction chromatography (HIC) on a phenyl sepharose column. Separate FSH and LH fractions were readily obtained in good yield by stepwise elution and the method can be easily adapted for large scale preparations of enriched fractions of eLH and eFSH. Three in vivo experiments were performed to evaluate the biological activity of fractions obtained by HIC. Experiment 1 showed that the biological activity of the $\mathrm{LH}$ fraction in inducing ovulation of preovulatory follicles was similar to that obtained with crude CEG on the basis of their respective immunological $\mathrm{LH}$ activities. This indicates that the $\mathrm{LH}$ bioactivity was not altered by HIC. Similarly, experiment 2 demonstrated that the biological activity of the FSH fraction was identical to that of CEG to superovulate mares, indicating that FSH activity was not altered by HIC. Final$I y$, experiment 3 revealed that ovarian responses with FSH were more homogeneous among animals and ovulations more synchronous than with CEG. 


\section{INTRODUCTION}

Dès 1930, la présence d'une gonadotropine sérique chez la jument gestante (pregnant mare serum gonadotropin, PMSG) a été décrite (Cole et Hart, 1930). Son activité mesurée sur la croissance ovarienne est détectée à partir du $37^{\mathrm{e}} \mathrm{j}$ de gestation jusqu'aux environs du $130^{\mathrm{e}} \mathrm{j}$. Cette hormone est d'origine placentaire (Allen et Moor, 1972) et par analogie avec son homologue humaine hCG, elle est maintenant dénommée eCG (equine chorionic gonadotropin).

Cette hormone équine a des propriétés physico-chimiques et biologiques tout à fait particulières. Ses propriétés biologiques les plus remarquables sont d'une part sa double activité folliculo-stimulante (FSH) et lutéinisante (LH) (Cole et al, 1946) et d'autre part, sa demi-vie exceptionnellement longue dans la circulation due à la structure particulière de ses chaînes oligosaccharidiques (Ward et Moore, 1979). Ces propriétés remarquables ont été et sont encore mises largement à profit chez les animaux domestiques (bovins, ovins, caprins, porcins) pour la synchronisation et l'induction d'ovulation ainsi que pour la superovulation. Par contre, toutes les tentatives effectuées avec eCG chez la jument dans le même but ont été vaines. Ainsi cette hormone présente une double activité $\mathrm{FSH}+\mathrm{LH}$ extrêmement importante chez toutes les espèces mais s'avère totalement inefficace dans sa propre espèce.

Cette propriété pour le moins inattendue a stimulé notre curiosité et nous avons décidé de rechercher l'origine structurelle des propriétés biologiques uniques de eCG. Après l'amélioration de la méthode de purification et l'étude des propriétés physico-chimiques (Combarnous et al, 1981), il nous est apparu qu'il était indispensable de comparer eCG aux gonado- tropines hypophysaires de la même espèce : eLH et eFSH.

Nous nous sommes donc attachés à la purification de ces hormones puis à l'étude de leur liaison aux récepteurs $\mathrm{LH}$ et $\mathrm{FSH}$, à la mise au point de dosages immunoenzymatiques spécifiques et enfin à l'étude de leur activité in vivo chez la jument.

\section{PURIFICATION DES GONADO- TROPINES HYPOPHYSAIRES ÉQUINES}

Puisque eCG est essentiellement utilisée chez les diverses espèces domestiques pour son activité folliculo-stimulante, nous nous sommes d'abord intéressés à la purification et à la caractérisation de la FSH équine (Combarnous et Hengé, 1981). La méthode de purification mise au point lors de cette étude est très lourde et la séparation des activités FSH et $\mathrm{LH}$ sur la base des charges des 2 hormones n'a pu être réalisée qu'après un très grand nombre d'étapes et en isolant chacune des différentes isoformes de la FSH équine. Nous avons ainsi pu obtenir les isoformes de eFSH à un très haut degré de pureté mais avec des rendements faibles. Ces préparations nous ont permis de caractériser de manière très approfondie cette hormone in vitro (Combarnous et al, 1984a) mais, en aucun cas l'étude de cette hormone n'était possible in vivo et en particulier chez la jument.

Plus tard, nous avons réalisé la purification de la LH équine (Guillou et Combarnous, 1983) en parallèle de celle de la FSH ainsi que sa caractérisation physicochimique mais pour les mêmes raisons que pour eFSH, les quantités obtenues n'ont pas permis d'aborder l'étude in vivo de cette hormone.

La difficulté de séparation de eFSH et eLH est due d'une part au fait que leurs 
poids moléculaires sont très proches et d'autre part, qu'elles présentent toutes 2, un important polymorphisme de charge et que les points isoélectriques des isoformes s'étalent dans la même gamme de $\mathrm{pH}$. II faut donc strier" chaque isoforme de eLH et de eFSH. D'autre part, la technique de précipitation fractionnée au sulfate d'ammonium ne permet pas de séparer eLH et eFSH à l'inverse des $\mathrm{LH}$ et FSH de nombreuses autres espèces.

Afin de séparer la LH et la FSH équines avec des rendements permettant leur utilisation chez la jument, il fallait donc utiliser une propriété physico-chimique qui ne soit ni leur taille ni leurs charges. Le développement de nouveaux gels chromatographiques permettant la séparation des protéines sur la base de leur hydrophobicité nous a donné l'opportunité de tenter la séparation de eLH et eFSH sur cette base.

La technique utilisée a été la chromatographie d'interaction hydrophobe (hydrophobic interaction chromatography; HIC) sur gel de phényle-sépharose (Pharmacia). $\dot{A}$ haute force ionique en présence d'un sel chaotropique (1,0 mol..- $\mathrm{I}^{-1}$ ammonium sulfate), les interactions polaires sont fortement affaiblies tandis que les interactions entre les groupements phényle du gel et les résidus hydrophobes des protéines sont favorisées. Dans ces conditions, la LH et la FSH d'un extrait hypophysaire brut équin sont toutes 2 retenues. L'établissement d'un gradient décroissant de force ionique (1->0 mol. I $^{-1}$ ammonium sulfate) concomitamment à un gradient croissant d'éthylène glycol ( $0 \rightarrow 50 \%)$ permet l'élution séquentielle des deux activités gonadotropes (Hofferer et al, 1992).

Bien sûr, à cette étape aucune des 2 gonadotropines n'est hautement purifiée mais l'essentiel pour leur utilisation in vivo est qu'elles soient séparées. Néanmoins, il est remarquable que cette méthode permet d'obtenir, en une seule étape, la $\mathrm{LH}$ équine à un degré de pureté déjà très élevé (environ 50\%).

Afin de rendre la méthode utilisable à l'échelle industrielle, nous avons utilisé des colonnes de tailles largement supérieures et nous avons recherché les paliers d'élution qui permettraient de supprimer le gradient. En effet cette approche oblige à mesurer les 2 activités dans les différentes fractions. Notre souhait était de définir des conditions où les 2 hormones seraient éluées dans 2 étapes successives facilement reproductibles.

La figure 1 montre que ce but a été atteint à la condition (prévisible) d'accepter une légère contamination de chaque hormone par l'autre.

La mise au point de cette méthode nous a permis de produire des quantités suffisantes de FSH et de LH équines semipurifiées pour leur utilisation chez la jument.

\section{SPÉCIFICITÉ DES GONADOTROPINES ÉQUINES}

La disponibilité de $\mathrm{FSH}$ et de $\mathrm{LH}$ équines hautement purifiées nous a permis d'étudier de manière plus systématique l'origine de la double activité LH+FSH de la CG équine (Combarnous et al, 1984b).

À cette fin, nous avons développé divers dosages radiorécepteurs (RRA) pour $\mathrm{LH}$ et $\mathrm{FSH}$, utilisant des membranes testiculaires de diverses espèces dont le cheval (Guillou et Combarnous, 1983).

Dans chacun de ces RRA, le traceur radio-iodé (125I-LH ou ${ }^{125} \mathrm{I}-\mathrm{FSH}$ ) était de même origine que les membranes testiculaires. Pour déterminer l'activité des hormones équines dans chacun de ces RRA, les courbes d'inhibition du traceur par ces hormones ont été comparées à celles obtenues avec les FSH et LH hautement pu- 


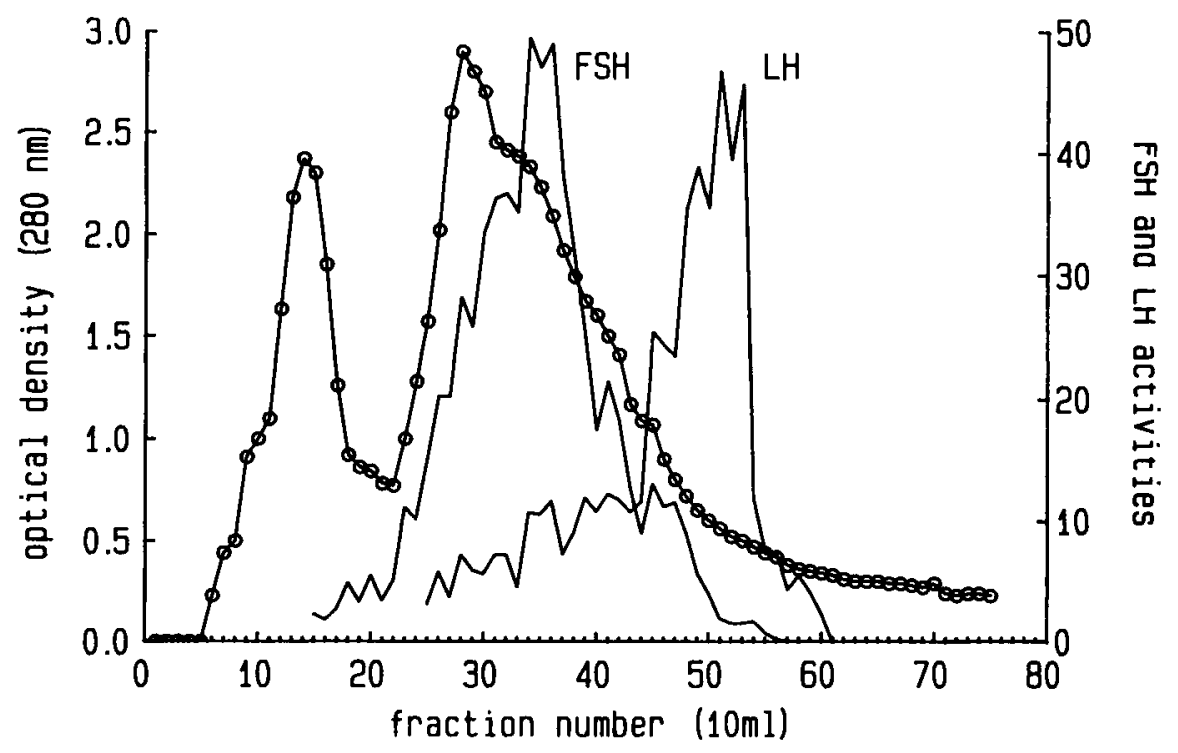

Fig 1. Séparation des gonadotropines hypophysaires équines (eLH et eFSH) par chromatographie d'interaction hydrophobe sur Phényl-Sépharose. L'extrait brut hypophysaire $(1 \mathrm{~g})$ a été dissous dans $50 \mathrm{ml}$ de tampon ammonium sulfate à $25 \%$ de saturation en ammonium bicarbonate 0,05 mol. $^{-1}$ puis chargé sur une colonne $(60 \mathrm{ml})$ de phényl-sépharose 4B (Pharmacia) équilibrée dans le même tampon. Un gradient linéaire de concentration de sulfate d'ammonium ( 25 à $0 \%$ de saturation) en tampon bicarbonate d'ammonium $0,05 \mathrm{mmol}^{-1} \mathrm{I}^{-1}$ a été établi entre les fractions 18 et 75 . Les activités $\mathrm{LH}$ et FSH des fractions ont été mesurées par dosages radio-immunologiques spécifiques.

rifiées de la même espèce. Dans ces conditions, il nous a été possible, pour chaque espèce, de mesurer l'activité LH et l'activité FSH des hormones équines par rapport à la $\mathrm{LH}$ et à la $\mathrm{FSH}$ de l'espèce considérée. De cette manière, nous avons pu réellement déterminer les rapports d'activités FSH/LH de ces hormones dans chaque espèce.

L'ensemble de nos résultats indiquent que eCG et eLH présentent dans les espèces non-équines étudiées, des activités de liaison similaires à celles des $\mathrm{LH}$ tandis que leurs activités FSH ne sont que de $20-30 \%$ de celles des FSH correspondantes. Ainsi dans tous ces cas, le rap- port des activités est largement en faveur des activités LH de 3 à 5 fois. Certains auteurs (Murphy et Martinuk, 1991) ont prétendu l'inverse mais sur la base de systèmes totalement hétérologues interdisant toute comparaison des rapports d'activité FSH/LH. À l'inverse de eCG et eLH, la eFSH ne présente que la seule activité $\mathrm{FSH}$, quelle que soit l'espèce utilisée pour le RRA.

Dans les RRA équins, la situation est beaucoup plus claire. En effet dans ce cas, eCG et eLH ne présentent strictement aucune activité FSH et cette dernière, comme dans les systèmes hétérologues, ne présente aucune activité LH. Ainsi, 
dans l'espèce équine comme dans l'espèce humaine, la choriogonadotropine secrétée par le placenta pendant une partie de la gestation possède exclusivement une activité $\mathrm{LH}$.

II doit cependant être souligné que l'activité de liaison aux récepteurs testiculaires équins à $\mathrm{LH}$ est beaucoup plus faible pour eCG que pour eLH (4\%) bien que les séquences d'acides aminés des 2 hormones soient strictement identiques. La eLH et la eCG semblent être les produits de l'expression non seulement des mêmes gènes $\alpha$ (sous-unité commune à toutes les hormones glycoprotéiques) mais aussi des mêmes gènes $B$ (sousunités spécifiques) par les cellules hypophysaires et placentaires respectivement. Par conséquent, la eCG est une eLH placentaire et ne diffère de la eLH hypophysaire que par la structure de ses chaînes oligosaccharidiques (Sugino et al, 1987). Ces chaînes de eCG ont donc, sur la liaison de l'hormone à ses récepteurs, un effet défavorable. Puisque eCG est plus facilement disponible que la eLH, un de nos projets est de modifier ses chaînes oligosaccharidiques afin de lui conférer la même activité de liaison que la eLH. II n'est pas certain que ce but soit compatible avec le maintien de sa demi-vie exceptionnellement longue.

Contrairement à eLH et eCG, les gonadotropines humaines hLH et hCG diffèrent assez fortement au niveau des séquences d'acides aminés de leurs sous-unités $B$. Elles ne sont donc évidemment pas les produits d'un même gène, mais conservent malgré tout une activité $\mathrm{LH}$ chez toutes les espèces. À l'inverse, eLH et eCG sont une seule et mème hormone, présentant deux activités chez les espèces non-équines. C'est en particulier l'étude du comportement de ces différentes hormones qui nous a conduit à développer le modèle de "spécificité négative" (Combarnous et Hengé, 1981).

\section{DOSAGES IMMUNOLOGIQUES DES GONADOTROPINES ÉQUINES}

La mise au point dans notre laboratoire de méthodes permettant l'obtention de eCG, eLH et eFSH hautement purifiées nous a conduit à mettre au point des dosages spécifiques et sensibles pour ces hormones. Dans un premier temps, des dosages radio-immunologiques très efficaces ont été mis au point (Cahoreau et Combarnous, 1987). Plus récemment, il est apparu nécessaire de disposer de dosages aussi performants mais moins contraignants, en termes de matériel et de sécurité, pour pouvoir être utilisés sur le terrain avec un équipement minimum. Nous ne décrirons donc succinctement ci-après que les dosages immuno-enzymatiques (ELISA) de ces hormones que nous avons mis au point.

\section{eCG}

Le dosage mis au point pour cette hormone est un ELISA compétition dans lequel de la eCG fixée aux puits de microtitration entre en compétition avec l'hormone en solution (inconnue ou standard) pour l'anticorps spécifique. Ainsi plus la concentration d'hormone en solution est importante et moins la quantité d'anticorps fixé à la eCG insolubilisée est élevée. Cet anticorps est détecté par un second anticorps anti-gammaglobulines de lapin conjugué à la peroxidase.

Ce dosage pratiquable sur le terrain en moins d'1 h permet, par exemple, aux producteurs de eCG/PMSG d'apprécier la concentration circulante de l'hormone et de n'effectuer le prélèvement sanguin sur la jument (gardée à l'attache pendant le dosage) que si cette concentration est suffisante. D'autre part, dans des conditions légèrement différentes, ce dosage est un 
test de gestation chez la jument au-delà du $37^{\mathrm{e}} \mathrm{j}$. Enfin, nos dosages immunologiques (RIA et ELISA) ont été soigneusement comparés aux dosages biologiques in vitro et in vivo de eCG et sont utilisés en routine pour le contrôle de nombreuses préparations commerciales. À cet effet, nous avons du sélectionner et caractériser notre propre standard de eCG car aucun standard international fiable de cette hormone n'existe.

\section{eLH}

Du fait de l'identité des chaînes polypeptidiques $\alpha$ et $\beta$ de eCG et eLH, cette dernière croise parfaitement dans les dosages immunologiques de eCG. Néanmoins, les concentrations circulantes de eLH sont considérablement inférieures à celles de eCG et la sensibilité exigée est largement supérieure. Pour ce qui concerne le RIA, il a été possible d'atteindre le seuil de détection ad hoc. Par contre, dans I'ELISA compétition, il n'est pas possible de suivre les concentrations basales de eLH. Nous tentons actuellement de mettre au point un dosage ELISA sandwich car cette approche est généralement plus sensible mais jusqu'à présent, nos tentatives ont été infructueuses.

\section{eFSH}

Le meilleur dosage immunologique de eFSH actuellement pratiqué au laboratoire est le dosage par immunochimioluminescence de hFSH (Amersham).

\section{ACTIVITÉ DES GONADOTROPINES ÉQUINES CHEZ LA JUMENT}

Nous avons indiqué plus haut que la eCG qui présente une double activité $\mathrm{FSH}+\mathrm{LH}$ chez les espèces non équines, est totalement dépourvue d'activité FSH chez la jument et ne présente qu'une activité LH faible. II semblerait que le rôle de eCG soit limité à provoquer l'ovulation des corps jaunes secondaires et à la stimulation temporaire de la production de progestérone par les différents corps jaunes (primaires et secondaires) jusqu'à ce que le placenta prenne le relais (Murphy et Martinuk, 1991).

À l'inverse de eCG, des préparations gonadotropes non équines (FSH porcine, hCG) sont actives chez la jument. Malheureusement, l'injection de gonadotropines hétérologues provoque, chez la jument, l'apparition rapide et intense d'anticorps neutralisant rendant inefficaces les injections ultérieures (Duchamp et al, 1987).

Pour ces raisons, il était important d'envisager l'utilisation des gonadotropines hypophysaires équines pour la maîtrise de la reproduction chez la jument. Celle-ci devęnait possible, comme nous l'avons vu, depuis que nous avions pu mettre au point une méthode semi-industrielle de séparation de eLH et eFSH à partir d'un extrait brut.

Les activités $\mathrm{LH}$ chez la jument de 25 $\mathrm{mg}$ d'extrait hypophysaire brut ou de $8 \mathrm{mg}$ de fraction III ( $2 \mathrm{mg}$ de eLH pure dans les 2 cas d'après ELISA) ont été suivies en ultrasonographie par la mesure du nombre de juments qui, possédant un follicule de plus de $35 \mathrm{~mm}$ de diamètre, ont ovulé dans les $48 \mathrm{~h}$ suivant l'injection. Dans le groupe contrôle, seules $7 / 25$ ont ovulé tandis que dans les groupes traités par l'extrait ou par la fraction III, les rapports ont été $24 / 25$ et $19 / 28$ respectivement. Dans le lot traité par la fraction III, 7 juments n'ont ovulé que 6 j après l'injection. Dans cette expérience préliminaire, la fraction III s'avère donc active mais peut être légèrement moins que l'extrait brut. Comme la quantité de eLH dans la fraction III est 
basée sur son activité immunologique, il se pourrait que le rapport $B / I$ dans cette fraction soit légèrement plus faible que dans l'extrait. Une autre hypothèse est qu'un facteur synergique de eLH soit présent dans l'extrait brut et absent dans la fraction III (FSH).

Les activités FSH de l'extrait hypophysaire et de la fraction II (eFSH) ont d'abord été appréciées par la mesure du nombre de follicules $>30 \mathrm{~mm}$ induits par jument. Les injections de doses de $25 \mathrm{mg}$ d'extrait brut ou de $10 \mathrm{mg}$ de fraction II $(1,25 \mathrm{mg}$ de eFSH pure dans les 2 cas) donnent la même réponse avec 3 follicules par jument au lieu de 1 dans le groupe contrôle. Les nombres d'ovulations induites par $25 \mathrm{mg}$ d'extrait (2 mg eLH pure) après traitements préalables par l'extrait ou la fraction II sont respectivement de $2,2 \pm 1,8$ et $2,1 \pm 1,2$ et ne sont donc pas significativement différents l'un de l'autre mais supérieurs au groupe contrôle $(1,0 \pm 0,0)$. L'intérêt de la fraction II semi-purifiée par rapport à l'extrait brut est de donner une moindre dispersion des nombres d'ovulations (0-3 au lieu de 0-6) et des moments d'ovulation en absence d'induction exogène par $\mathrm{LH}$.

Nos résultats montrent clairement que les préparations de gonadotropines hypophysaires équines sont efficaces dans la maîtrise de la fonction ovarienne chez la jument. Nos premiers résultats n'indiquent pas que la séparation de eLH et eFSH améliore quantitativement l'activité de chacune des 2 hormones pour ses indications propres. Cependant, une plus grande homogénéité des réponses est obtenue avec les hormones séparées qu'avec l'extrait brut. De plus, les doses de fractions II et III à utiliser pour les différentes indications devraient pouvoir être encore optimisées. Enfin, grâce à l'excellent rendement de la préparation des 2 fractions tant en terme pondéral que d'activité, cette séparation est intéressante car elle permet de presque doubler le nombre total de doses ( $\mathrm{LH}$ ou FSH) préparées à partir d'un même lot d'hypophyses. Le coût actuel des hypophyses équines justifie, à lui seul, d'effectuer la séparation des 2 hormones. De plus, cette séparation est nécessaire pour mieux appréhender les rôles respectifs des deux hormones et optimiser les doses de chacune d'elles. Enfin, d'autres hormones et facteurs pourront également être plus facilement purifiés et exploités à partir de ces fractions qu'à partir de l'extrait brut.

\section{REMERCIEMENTS}

Les auteurs remercient la région Centre, le ministère de la Recherche et de la Technologie et le Critt-ISIS pour leur soutien dans ce travail.

\section{RÉFÉRENCES}

Allen W R, Moor R M (1972) The origin of the equine endometrial cups. 1. Production of PMSG by fetal trophoblast cells. J Reprod Fertil 29, 313-316

Cahoreau C, Combarnous $Y$ (1987) Comparison of two reference preparations for horse chorionic gonadotrophin in four in vivo and in vitro assays. J Reprod Fertil 79, 281-287

Cole HH, Hart GH (1930) The potency of blood serum of mares in progressive stages of pregnancy in effecting the sexual maturity of the immature rat. Am J Physiol 93, 57-62

Cole HH, Penchars RL, Goss H (1946) On the biological properties of highly purified gonadotrophin from pregnant mare serum. Endocrinology $27,548-553$

CombarnousY, Guillou F, Martinat N (1984a) Comparison of in-vitro follicle-stimulating hormone (FSH) activity of equine gonadotropins (luteinizing hormone, FSH, and chorionic gonadotropin) in male and female rats. Endocrinology $115,1821-1827$

CombarnousY, Guillou F, Martinat N, Cahoreau C (1984b) Origine de la double activité FSH + LH de la choriogonadotropine équine (eCG/PMSG). Ann Endocrinol 45, 261-268 
Combarnous $Y$, Hengé M-H (1981) Equine follicle-stimulating hormone. Purification, acid dissociation and binding to equine testicular tissue. J Biol Chem 256, 9567-9572

Combarnous Y, Salesse R, Garnier J (1981) Physicochemical properties of pregnantmare serum gonadotropin. Biochim Biophys Acta 667, 267-276

Duchamp G, Bour B, Combarnous $\mathrm{Y}$, Palmer E (1987) Alternative solutions to hCG induction of ovulation in the mare. J Reprod Fertil 35, 221-228

Guillou F, Combarnous Y (1983) Purification of equine gonadotropins and comparative study of their acid-dissociation and receptorbinding specificity. Biochim Biophys Acta 735, 229-236
Hofferer S, Lecompte F, Magallon T, Palmer E, Combarnous $Y(1992)$ Equine luteinizing hormone and follicle-stimulating hormone: separation, immunoassays and use for ovulation induction and superovulation in mares. $J \mathrm{Re}$ prod Fertil (accepté pour publication)

Murphy BD, Martinuk S D (1991) Equine chorionic gonadotropin. Endocrine Rev 12, 27-44

Sugino $H$, Bousfield G R, Moore W T, Ward DN (1987) Structural studies on equine glycoprotein hormones. J Biol Chem 262, 8610-8615

Ward DN, Moore WT (1979) Comparative study of mammalian glycoprotein hormones. In: Animal models for research in fertility and contraception (Alexander $\mathrm{J}$ ed) Harper and Row, Baltimore, MD, 151 